\title{
Minimizing Symbol Error Rate for Cognitive Relaying with Opportunistic Access $\diamond$
}

\author{
Ammar Zafar*, Mohamed-Slim Alouini*, Yunfei Chen ${ }^{\star}$, Redha M. Radaydeh* \\ *Electrical Engineering Program, King Abdullah University of Science and Technology (KAUST) \\ Thuwal, Makkah Province, Saudi Arabia \\ Emails:\{ammar.zafar, slim.alouini, redha.radaydeh\}@kaust.edu.sa \\ ${ }^{\star}$ School of Engineering, University of Warwick \\ Coventry, UK, CV4 7AL \\ Email: yunfei.chen@warwick.ac.uk
}

\begin{abstract}
In this paper, we present an optimal resource allocation scheme (ORA) for an all-participate (AP) cognitive relay network that minimizes the symbol error rate (SER). The SER is derived and different constraints are considered on the system. We consider the cases of both individual and global power constraints, individual constraints only and global constraints only. Numerical results show that the ORA scheme outperforms the schemes with direct link only and uniform power allocation (UPA) in terms of minimizing the SER for all three cases of different constraints. Numerical results also show that the individual constraints only case provides the best performance at large signal-to-noise-ratio (SNR).

Index Terms-Cognitive relays, opportunistic access, optimal resource allocation, symbol error rate
\end{abstract}

\section{INTRODUCTION}

In a cognitive radio network, the unlicensed or secondary users communicate using time and space intervals, called "spectral holes", during which the licensed or primary users are not active [1]. The transmitting secondary user can use other secondary users as relays to achieve diversity gain, which is cognitive relaying [2]-[4]. In cognitive relaying, after detecting a "spectral hole", the source broadcasts the signal to the destination and the relays. The relays then forward the signal to the destination.

The secondary user has to ensure that minimum interference is caused to the primary user. However, it also has to ensure that it achieves optimum throughput and symbol error rate (SER). These seemingly conflicting objectives have been the subject of much work in recent years. However, a majority of the recent works focuses on optimizing the throughput. These works also assume an "underlay" setup, in which the primary and secondary networks co-exist. References [5]-[12], all discuss optimal power allocations strategies for throughput maximization for relay assisted cognitive radio networks in an "underlay" setup.

In this paper, a cognitive relay network with opportunistic access to the licensed bands is considered. The objective here is to minimize the SER of the transmitting user while maintaining the throughout at an "acceptable" level. This 'acceptable'

\footnotetext{
${ }^{\diamond}$ This work was funded by King Abdullah University of Science and Technology (KAUST).
}

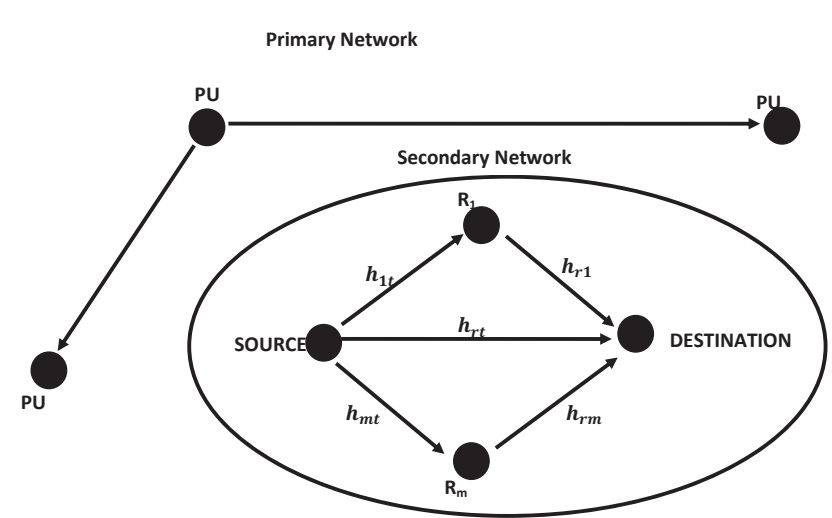

Fig. 1: Primary and secondary networks.

level depends on the application and the system requirements and can differ for different systems. To reduce complexity at the relays, only the source performs spectrum sensing. The optimization problem is considered under different constraints. Full channel state information (CSI) is assumed at the central controller, who performs the optimization and informs the source and the relays about their allotted power through feedback channels. We consider amplify-and-forward (AF) relaying.

Numerical results show the dependence of the optimal sensing time on the primary user's signal-to-noise-ratio (SNR). Results also show that the system achieves a lower SER in the case of a global power constraint only as compared to the system under both global and individual power constraints.

\section{SySTEM MOdEL}

Consider a secondary network in which a source node transmits data to a destination node through the help of $m$ relays with access to the licensed bands only available in the absence of primary activity. The source and the relays transmit on orthogonal channels; therefore, do not cause interference with each other. A time orthogonal system is assumed in the following. After the system starts up, during the first time slot, the source searches for a "spectral hole". The source transmits data to the destination and the $m$ relays, on detection of a "spectral hole". Otherwise it remains silent. The relays, each in a different time slot, amplify-and-forward the signal to the 
destination. Hence, one packet of data consumes $m+1$ time slots for transmission.

\section{A. Received Signal Model}

With such a mode of operation, there can be two received signal models.

1) Without Interference from the Primary User: This is the case when, with probability $1-P_{f}$ where $P_{f}$ is the probability of false alarm, the source correctly detects no primary activity. The signals received at the destination and the relays from the source are

$$
\begin{aligned}
& y_{r_{t}}=\sqrt{E_{S T}} h_{r_{t}} s+n_{r_{t}}, \\
& y_{i_{t}}=\sqrt{E_{S T}} h_{i_{t}} s+n_{i_{t}},
\end{aligned}
$$

where $s$ is the zero-mean and unit-energy transmitted symbol, $E_{S T}$ is the source energy, $h_{r_{t}}$ is the channel response between the source and the destination, $h_{i_{t}}$ is the channel response between the $i$ th relay and the source and $n_{r_{t}} \sim C N\left(0, \sigma_{n_{r_{t}}}^{2}\right)$ and $n_{i_{t}} \sim C N\left(0, \sigma_{n_{i_{t}}}^{2}\right)$ are the complex Gaussian noise samples. The relay, after normalization, forwards the received signal to the destination. Therefore the received signal at the destination from the $i$ th relay is

$$
y_{r_{i}}=\sqrt{E_{i}} h_{r_{i}} s_{i}+n_{r_{i}}
$$

where $h_{r_{i}}$ is the known channel response between the receiver and the $i$ th relay, $E_{i}$ is the $i$ th relay's energy and $n_{r_{i}} \sim$ $C N\left(0, \sigma_{n_{r_{i}}}^{2}\right)$ is the complex Gaussian noise. Substituting $s_{i}$ in (3) gives

$$
y_{r_{i}}=\sqrt{\frac{E_{S T} E_{i}}{E_{S T}\left|h_{i_{t}}\right|^{2}+\sigma_{n_{i_{t}}}^{2}}} h_{r_{i}} h_{i_{t}} s+\tilde{n_{r_{i}}},
$$

where $\tilde{n_{r_{i}}} \sim C N\left(0, \tilde{\sigma_{n_{r_{i}}}^{\tilde{2}}}\right)$ and

$$
\sigma_{n_{r_{i}}}^{\tilde{2}}=\frac{E_{i}\left|h_{r_{i}}\right|^{2} \sigma_{n_{i_{t}}}^{2}}{E_{S T}\left|h_{i_{t}}\right|^{2}+\sigma_{n_{i_{t}}}^{2}}+\sigma_{n_{r_{i}}}^{2} .
$$

Writing the $m+1$ received signals in matrix form, one has

$$
\mathbf{y}=\mathbf{h} s+\mathbf{n}
$$

where

$$
\begin{gathered}
\mathbf{y}=\left[\frac{1}{\sigma_{n_{r_{t}}}} y_{r_{t}} \frac{1}{\sigma_{n_{r_{1}}}^{\tilde{2}}} y_{r_{1}} \ldots \ldots \ldots \frac{1}{\sigma_{n_{r_{m}}}} y_{r_{m}}\right]^{T} \\
\mathbf{h}=\left[\sqrt{\frac{E_{S T}}{\sigma_{n_{r_{t}}}^{2}}} h_{r_{t}} \sqrt{\frac{E_{S T} E_{1}}{\sigma_{n_{r_{1}}}^{\tilde{2}}\left(E_{S T}\left|h_{1_{t}}\right|^{2}+\sigma_{n_{1_{t}}}^{2}\right)}} h_{r_{1}} h_{1_{t}} \ldots\right. \\
\left.\sqrt{\frac{E_{S T} E_{m}}{\sigma_{n_{r_{m}}}^{2}\left(E_{S T}\left|h_{m_{t}}\right|^{2}+\sigma_{n_{m_{t}}}^{2}\right)}} h_{r_{m}} h_{m_{t}}\right]^{T}
\end{gathered}
$$

and $\mathbf{n} \sim C N(\mathbf{0}, \mathbf{I})$

2) With Interference from the Primary User: In this case, with probability $1-P_{d}$ where $P_{d}$ is the probability of detection, the source misses the transmission from the primary user and transmits, which causes interference. The signals at the destination from both the source and the relays now also include a interfering signal due to primary user activity

$$
\begin{gathered}
y_{r_{t}}=\sqrt{E_{S T}} h_{r_{t}} s+n_{r_{t}}+y_{I_{r_{t}}} \\
y_{i_{t}}=\sqrt{E_{S T}} h_{i_{t}} s+n_{i_{t}}+y_{I_{i_{t}}}
\end{gathered}
$$

where $y_{I_{r_{t}}}$ and $y_{I_{i_{t}}}$ are the interference signals. Taking into account the fact that the source and relays have no knowledge of the interfering signal and adopting the same approach as previously, one can write

$$
y_{r_{i}}=\sqrt{\frac{E_{S T} E_{i}}{E_{S T}\left|h_{i_{t}}\right|^{2}+\sigma_{n_{i_{t}}}^{2}}} h_{r_{i}} h_{i_{t}} s+\hat{n_{r_{i}}}+y_{\hat{I}_{r_{i}}}
$$

where $\hat{n_{r_{i}}} \sim C N\left(0, \hat{\sigma_{n_{r_{i}}}}\right)$ and

$$
\sigma_{n_{r_{i}}}^{\hat{2}}=\frac{E_{i}\left|h_{r_{i}}\right|^{2} \sigma_{n_{i_{t}}}^{2}}{E_{S T}\left|h_{i_{t}}\right|^{2}+\sigma_{n_{i_{t}}}^{2}}+\sigma_{n_{r_{i}}}^{2}
$$

and

$$
\hat{y}_{i_{i}}=y_{I_{r_{i}}}+\sqrt{\frac{E_{i}}{E_{S T\left|h_{i_{t}}\right|^{2}+\sigma_{n_{i_{t}}}^{2}}}} h_{r_{i}} y_{I_{i_{t}}}
$$

Again in matrix form one has

$$
\mathbf{y}_{I}=\mathbf{h}_{I} s+\mathbf{n}_{I}+\mathbf{Y}_{I}
$$

where

$$
\mathbf{y}_{I}=\left[\frac{1}{\sigma_{n_{r_{t}}}} y_{r_{t}} \frac{1}{\sigma_{n_{r_{1}}}} y_{r_{1}} \ldots \ldots \ldots \frac{1}{\sigma_{n_{r_{m}}}} y_{r_{m}}\right]^{T}
$$

$$
\begin{aligned}
& \mathbf{h}_{I}=\left[\sqrt{\frac{E_{S T}}{\sigma_{n_{r_{t}}}^{2}}} h_{r_{t}} \quad \sqrt{\frac{E_{S T} E_{1}}{\sigma_{n_{r_{1}}}^{2}\left(E_{S T}\left|h_{1_{t}}\right|^{2}+\sigma_{n_{1_{t}}}^{2}\right)}} h_{r_{1}} h_{1_{t}} \ldots\right. \\
& \left.\sqrt{\frac{E_{S T} E_{m}}{\sigma_{n_{r_{m}}}^{2 \hat{}}\left(E_{S T}\left|h_{m_{t}}\right|^{2}+\sigma_{n_{m_{t}}}^{2}\right)}} h_{r_{m}} h_{m_{t}}\right]^{T} \\
& \mathbf{Y}_{I}=\left[\frac{1}{\sigma_{n_{r_{t}}}} y_{I_{r_{t}}} \frac{1}{\sigma_{n_{r_{1}}}} \hat{y}_{I_{1}} \ldots \ldots \cdot \frac{1}{\sigma_{n_{r_{m}}}} y_{I_{m}}\right]^{T}
\end{aligned}
$$

and $\mathbf{n}_{I} \sim C N(\mathbf{0}, \mathbf{I})$.

B. Spectrum Sensing is performed, by means of an energy detector, for the first $t_{s}$ seconds out of total time slot duration of $T$ seconds at the source node only. The remaining $T-t_{s}$ is used for transmission, after detecting a "spectral hole". The probabilities of detection and false alarm, according to [13], are given by

$$
\begin{gathered}
P_{d}=Q\left(\frac{\lambda-N-\gamma_{d}}{\sqrt{2\left(N+2 \gamma_{d}\right)}}\right) \\
P_{f}=Q\left(\frac{\lambda-N}{\sqrt{2 N}}\right)
\end{gathered}
$$

respectively, where $\lambda$ is the threshold of the energy detector, $N=t_{s} f_{s}$ is the number of samples, $f_{s}$ is the sampling frequency, $\gamma_{d}$ equals $N$ times the SNR at the output of the 
detector and $Q($.$) is the Gaussian Q$-function.

\section{OPTIMIZATION}

In this section, we formulate the optimization problem. We derive the SER first and then give the constraints on the optimization problem. Assuming maximal ratio combining (MRC) at the destination, the SER is

$$
\begin{aligned}
S E R=P\left(H_{0}\right)\left(Q\left(\sqrt{k \gamma_{0}}\right)\right)\left(1-P_{f}\right)+ \\
P\left(H_{1}\right)\left(Q\left(\sqrt{k \gamma_{I}}\right)\right)\left(1-P_{d}\right)
\end{aligned}
$$

where $\gamma_{0}$ is as the SNR after combining, $\gamma_{I}$ is the signalto-interference-noise-ratio (SINR) after combining, $k$ is a constant which depends on the modulation scheme used, $P\left(H_{0}\right)$ is the probability of no primary user transmission and $P\left(H_{1}\right)=1-P\left(H_{0}\right)$ is the probability of a primary user transmission. The SNR $\gamma_{0}$ can be found as

$$
\gamma_{0}=\alpha+\sum_{i=1}^{m} \frac{\beta_{i}}{\sigma_{n_{r_{i}}}^{\tilde{2}}}
$$

where

$$
\alpha=\frac{p_{S T} T_{s}\left|h_{r_{t}}\right|^{2}}{\sigma_{n_{r_{t}}}^{2}} \quad \beta_{i}=\frac{p_{S T} p_{i} T_{s}^{2}\left|h_{r_{i}}\right|^{2}\left|h_{i_{t}}\right|^{2}}{p_{S T} T_{s}\left|h_{i_{t}}\right|^{2}+\sigma_{n_{i_{t}}}^{2}} .
$$

Note that we have replaced the source and relay energies with

$$
E_{S T}=p_{S T} T_{s} \quad \text { and } \quad E_{i}=p_{i} T_{S}
$$

where $p_{S T}$ and $p_{i} \mathrm{~s}$ are the source and relay powers, respectively and $T_{s}$ is the symbol time. Similarly, $\gamma_{I}$ can be expressed as

$$
\gamma_{I}=\frac{\left(\alpha+\sum_{i=1}^{m} \frac{\beta_{i}}{\sigma_{n_{r_{i}}}^{\hat{2}}}\right)^{2}}{\alpha+\sum_{i=1}^{m} \frac{\beta_{i}}{\sigma_{n_{r_{i}}}^{2}}+\frac{\alpha c}{\sigma_{n_{r_{t}}}^{2}}+\sum_{i=1}^{m} \frac{d_{i}}{\sigma_{n_{r_{i}}}^{2}} \frac{\beta_{i}}{\sigma_{n_{r_{i}}}^{\hat{2}}}}
$$

where

$$
\begin{aligned}
& c=E\left[\left|y_{I_{r_{t}}}\right|^{2}\right] \\
& d_{i}=E\left[\left|\hat{y}_{i}\right|^{2}\right]
\end{aligned}
$$

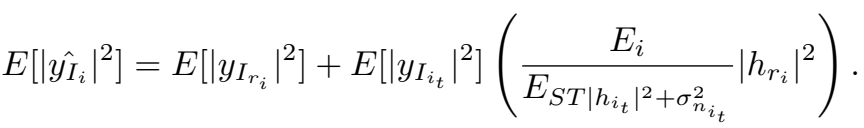

Substituting (11) and (12) in (10) gives the SER as

$$
\begin{aligned}
& S E R=P\left(H_{0}\right) Q\left(\sqrt{k\left(\alpha+\sum_{i=1}^{m} \frac{\beta_{i}}{\sigma_{n_{r_{i}}}^{\tilde{2}}}\right)}\right) \times \\
& \left(1-Q\left(\frac{\lambda-N}{\sqrt{2 N}}\right)\right)+P\left(H_{1}\right)\left(1-Q\left(\frac{\lambda-N-\gamma_{d}}{\sqrt{2\left(N+2 \gamma_{d}\right)}}\right)\right) \\
& \times Q\left(\sqrt{\frac{k\left(\alpha+\sum_{i=1}^{m} \frac{\beta_{i}}{\sigma_{n_{r_{i}}}^{2}}\right)^{2}}{\alpha+\sum_{i=1}^{m} \frac{\beta_{i}}{\sigma_{n_{r_{i}}}^{2}}+\frac{\alpha c}{\sigma_{n_{r_{t}}}^{2}}+\sum_{i=1}^{m} \frac{d_{i}}{\sigma_{n_{r_{i}}}^{2}} \frac{\beta_{i}}{\sigma_{n_{r_{i}}}^{2}}}}\right)
\end{aligned}
$$

Now we form the different constraints on the problem. First we consider both individual power constraints at the source and the relay and a global power constraint on the whole system. Therefore, the constraints are given by

$$
\begin{aligned}
& 0 \leq p_{S T} \leq p_{T}, 0 \leq p_{i} \leq p_{i}^{\max }, P_{f} \leq P_{f}^{t h} \\
& p_{S T}+\sum_{i=1}^{m} p_{i} \leq p_{\text {total }}, \quad \text { and } R T_{s}+t_{s} \leq T .
\end{aligned}
$$

where $p_{S T}$ is the power available at the source, $p_{i}^{\max }$ is the power available at each relay, $p_{\text {total }}$ is the power available to the whole system and $P_{f}^{t h}$ specifies the constraint on the probability of false alarm. The constraints on $P_{f}, T_{s}$ and $t_{s}$ are introduced to maintain an acceptable throughput. Next the two cases global power constraint only and individual power constraints only are considered. For the case of global power constraint only, the constraints will be

$P_{f} \leq P_{f}^{t h}, \quad p_{S T}+\sum_{i=1}^{m} p_{i} \leq p_{\text {total }}$, and $R T_{s}+t_{s} \leq T$.

In the other scenario, the constraints are given by

$$
\begin{gathered}
0 \leq p_{S T} \leq p_{T}, 0 \leq p_{i} \leq p_{i}^{\max }, P_{f} \leq P_{f}^{t h} \\
\text { and } R T_{s}+t_{s} \leq T
\end{gathered}
$$

A special case of importance is the absence of the direct link between source and destination, because the relays take on a more prominent role in the presence of no direct link. In this case, the SER can be obtained by replacing $\alpha=0$ in (13).

The joint optimization problem (13) under all three constraints cases, (14), (15) and (16), in non convex and hence, it is difficult to obtain closed-form expressions for the optimal solutions. Therefore, one has to resort to numerical techniques to find the optimal solution.

\section{NumericAl RESUlts}

Some selected numerical results are provided in this section. All noise variances are set at $1 . p_{T}$ and $p_{i}^{\max }$ are set at 2 , while $p_{\text {total }}$ is set at $2+1.5 \mathrm{~m}$. The total frame duration $T$ is set as $100 \mathrm{~ms}$. We set $P_{f}^{t h}=0.1$, and $R=100$. Binary Phase Shift Keying (BPSK) is the constellation considered. As the sensing time and the number of samples have a linear relationship, the results are given in terms of the number of samples, N. Figure 2 shows the SER as a function of the number of samples for different values of primary user's SNR, $\gamma$. It is evident from the figure that there is an optimal value of the number of samples at which the SER is minimized. This trade-off is due to the fact that with increase in $N$, the probability of detection, $P_{d}$, increases, hence, decreasing SER. However, an increase in $N$ means a decrease in symbol time, $T_{s}$, which causes $\gamma_{0}$ and $\gamma_{I}$ to decrease, which in turn increases SER. Therefore, a trade-off is seen in figure 2 . The primary user's, $\gamma$, greatly affects the optimal number of samples. When $\gamma$ is higher, it takes less time to achieve the same $P_{d}$ as opposed to, when $\gamma$ is lower. Hence, the smaller the $\gamma$, greater is the optimal number of samples. 


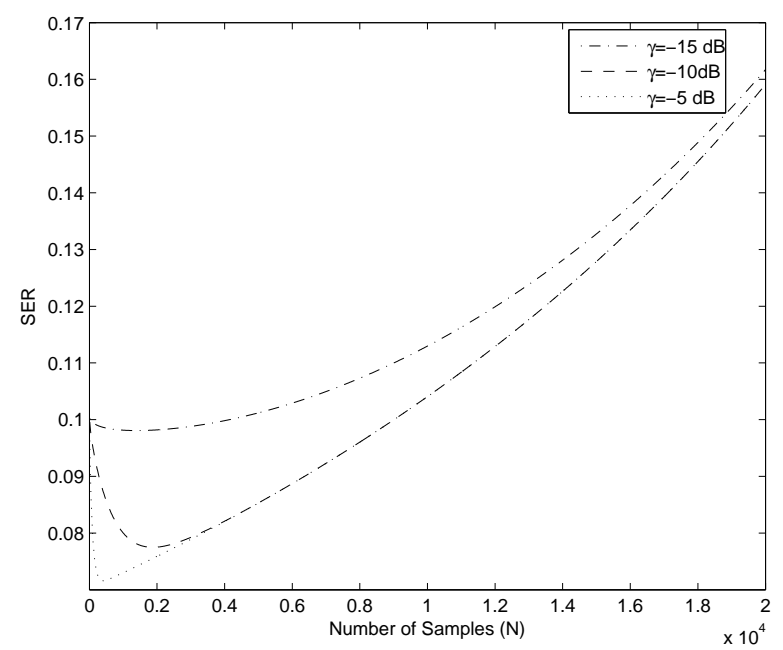

Fig. 2: SER as a function number of samples $(\mathrm{N})$.

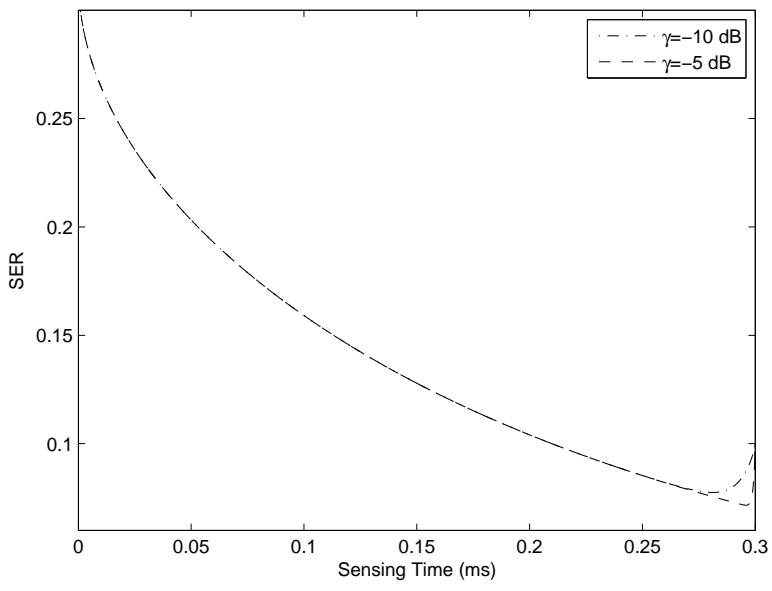

Fig. 3: SER as a function of the symbol time $\left(T_{s}\right)$

Figure 3 shows the SER plotted against the symbol time, $T_{s}$. As one can see, it has the opposite characteristic as in the case of optimal number of samples. This is because of the inverse relationship between the sensing time and symbol as expressed in the constraint $R T_{s}+t_{s} \leq T$. Therefore, the optimal value of the symbol time increases with increasing $\gamma$.

To analyze the performance of the optimization schemes as a function of the system SNR, we let $\sigma_{n_{r_{t}}}^{2}=\sigma^{2}$, $\sigma_{n_{i_{t}}}^{2}=\rho \sigma^{2}$ and $\sigma_{n_{r_{i}}}^{2}=\mu \sigma^{2}$, where $\rho$ and $\mu$ are constants which are set to 1 and then $\gamma_{s}=\frac{1}{\sigma^{2}}$. Figure 4 shows the performance of the various schemes as a function of $\gamma_{s}$. The figure shows that three all-participate optimal resource allocation(AP-ORA) with direct link scenarios, i.e. global constraint only (Gl), individual constraints only (Ind) and both global and individual constraints, far outstrip the cases of direct link only and uniform power allocation (UPA). In the no direct link (NDL) scenario, AP-ORA-NDL performs better under all three constraints than uniform power allocation with no direct link (UPA-NDL). However, for lower $\gamma_{s}$ the AP-

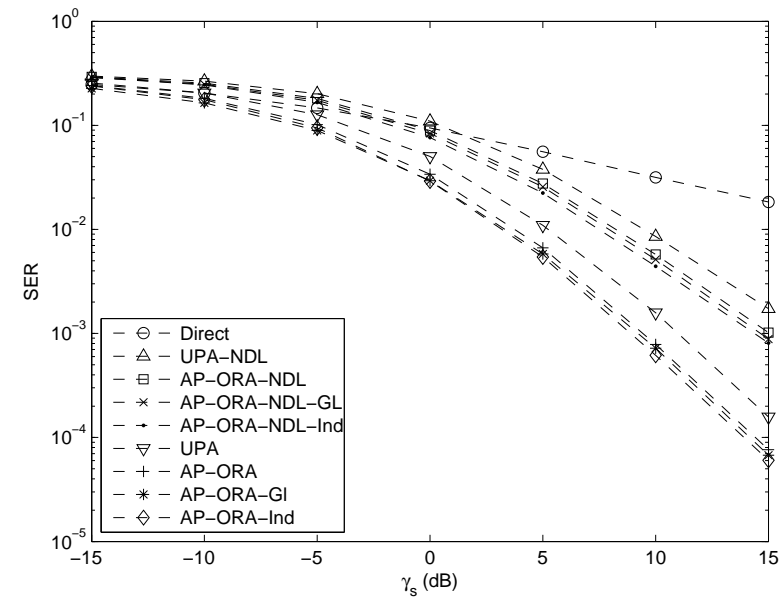

Fig. 4: Comparison of SER under different schemes and constraints.

ORA-NDL schemes provide greater SER than the direct link only case. This shows the significance of a robust direct link, particularly for lower $\gamma_{s}$. This fact is also evident by the difference in performance between the no direct link cases and the direct link scenarios. Among the different constraints, the global constraints only scenario outperforms the case with both global and individual constraints only, both in the direct and no direct link cases. This is because that even though the global constraint is same for both cases, the global only case is not limited by the individual constraints, hence more power can be allocated to the source or relay which has the best conditions for data transmission. Therefore, taking full advantage of the channel conditions and achieving a lower SER. The comparison between global constraint only and individual constraints only need further discussion.

The no direct link scenario is considered first. In this scenario, the individual constraints only case outperforms the global constraint only case for all values of $\gamma_{s}$. The difference between the two cases becomes more prominent at higher $\gamma_{s}$. The individual constraints only case gives lower SER than global constraints only because, even though in the latter scenario more power can be allocated to the relay with better channel, the total power available to the former is greater. And this larger power makes up for the fact that each relay has constrained power. In the direct link case, the global constraints only case gives better SER performance than the individual constraints only case for lower $\gamma_{s}$. This is due to the presence of a direct link. As discussed previously, the direct link has a great influence. The power of the direct link is limited in the individual case, however, more power can be allocated to the direct link in the global case. Therefore, even though the individual case has more total power, the global constraints only case outperforms it by allocating more power to the direct link. The individual constraints only case outperforms the global constraints case for higher $\gamma_{s}$. This is due to the fact that now there is less noise in the system and as there is more total power available in the individual case, it overcomes the limitation of the constrained source power and gives better SER. 
TABLE I: Glossary

\begin{tabular}{|c|c|}
\hline ACRONYM & FULL NAME \\
\hline AP-ORA & All-Participate with Optimal Resource Allocation \\
\hline AP-ORA-GL & All-PARTicipate Optimal Resource Allocation with Global CONSTRAint only \\
\hline UPA & UNIFORM POWER ALLOCATION \\
\hline AP-ORA-NDL & All-Participate Optimal Resource Allocation with no direct link \\
\hline AP-ORA-NDL-Ind & All-Participate Optimal Resource Allocation with IndiVidual CONSTRaints ONLY and NO DiRECT LinK \\
\hline UPA-NDL & UNIFORM POWER ALLOCATION WITH NO DIRECT LINK \\
\hline
\end{tabular}

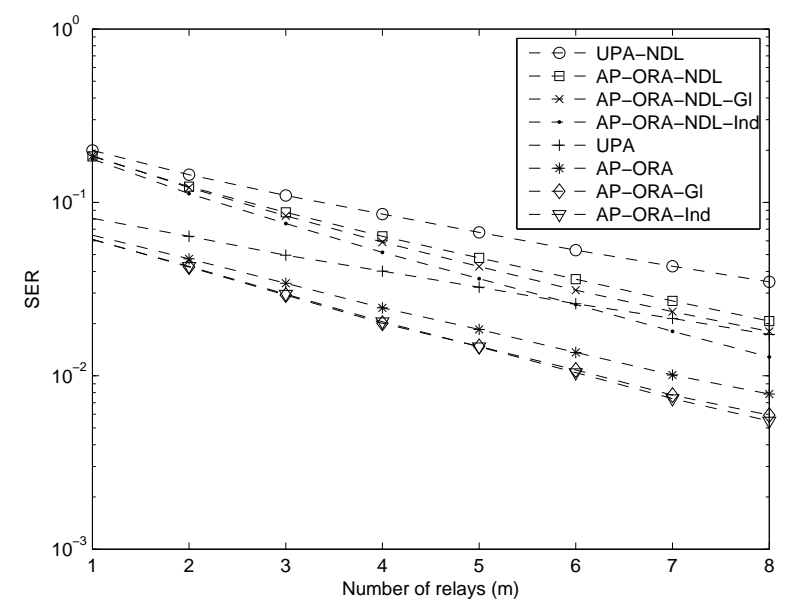

Fig. 5: SER as a function of number of relays (m).

The same phenomena can be observed in Figure 5, but this time as a function of the number of relays $(\mathrm{m})$. Again, the AP-ORA scenarios outperform their UPA counterparts. Additionally, for a larger number of relays the AP-ORA-NDL cases give lower SER than UPA. AP-ORA, with and without direct link, with both global and individual power constraints gives a higher SER than AP-ORA-Gl and AP-ORA-Ind, again with and without direct link. In the no direct link case APORA-NDL-Ind gives considerable better performance than AP-ORA-NDL-Gl, particularly for a large number of relays as AP-ORA-NDL-Ind has more total power. However, again it can be seen that, in the presence of a direct link, AP-ORAInd and AP-ORA-Gl are much closer in performance. And AP-ORA-Ind starts outperforming AP-ORA-Gl only when the number of relays is larger.

\section{CONCLUSions}

In this paper, an all-participate (AP) optimal resource allocation (ORA) that minimizes SER was proposed. It was shown that the AP-ORA scheme outstripped the conventional uniform power allocation (UPA) in terms of SER performance. The importance of the direct link to the system performance was also shown. It was also demonstrated that the AP-ORA-Ind outperformed both AP-ORA and AP-ORA-Gl for higher $\gamma_{s}$ and greater number of relays.

\section{REFERENCES}

[1] S. Haykin, "Cognitive radio: brain-empowered wireless communications," IEEE Journal on Selected Areas in Communications, vol. 23, no. 2, pp. 201-220, Feb. 2005.

[2] O. Simeone, J. Gambini, Y. Bar-Ness, and U. Spagnolini, "Cooperation and cognitive radio," in Proc. IEEE International Conference on Communications 2007 (ICC 2007), Glasgow, Scotland, UK, Jun. 2007, pp. 6511-6515.

[3] Y. Zou, Y. Yao, and B. Zheng, "Cognitive transmissions with multiple relays in cognitive radio networks," IEEE Transactions on Wireless Communications, vol. 10, no. 2, pp. 648-659, Feb. 2011.

[4] X. Mao and P. Qiu, "Cognitive relay," in Proc. of the Future Generation Communication and Networking (FGCN 2007), vol. 2, Jeju Island, Korea, Dec. 2007, pp. 264-269.

[5] W. Yue, B. Zheng, and Q. Meng, "Optimal power allocation for cognitive relay networks," in Proc. International Conference on Wireless Communications and Signal Processing 2009 (WCSP 2009), Nanjing, China, Nov. 2009, pp. 1-5.

[6] L. Li, X. Zhou, H. Xu, G. Y. Li, D. Wang, and A. Soong, "Simplified relay selection and power allocation in cooperative cognitive radio systems," IEEE Transactions on Wireless Communications, vol. 10, no. 1, pp. 33-36, Jan. 2011.

[7] X. Liu, B. Zheng, J. Cui, and W. Ji, "A new scheme for power allocation in cognitive radio networks based on cooperative relay," in Proc. 12th IEEE International Conference on Communication Technology 2010 (ICCT 2010), Tsukuba Science City, Ibaraki, Japan, Nov. 2010, pp. 861864.

[8] J. Mietzner, L. Lampe, and R. Schober, "Distributed transmit power allocation for multihop cognitive-radio systems," IEEE Transactions on Wireless Communications, vol. 8, no. 10, pp. 5187-5201, Oct. 2009.

[9] Z. Liu, Y. Xu, D. Zhang, and S. Guan, "An efficient power allocation algorithm for relay assisted cognitive radio network," in Proc. International Conference on Wireless Communications and Signal Processing 2010 (WCSP 2010), Suzhou, China, Oct. 2010, pp. 1-5.

[10] X. Qiao, Z. Tan, S. Xu, and J. Li, "Combined power allocation in cognitive radio-based relay-assisted networks," in Proc. IEEE International Conference on Communications Workshops 2010 (ICC 2010), Cape Town, South Africa, May 2010, pp. 1-5.

[11] X. Liu, B. Zheng, and W. Ji, "Cooperative relay with power control in cognitive radio networks," in Proc. 6th International Conference on Wireless Communications Networking and Mobile Computing 2010 (WiCOM 2010), Chengdu, China, Sep. 2010, pp. 1-5.

[12] L. Jayasinghe and N. Rajatheva, "Optimal power allocation for relay assisted cognitive radio networks," in IEEE 72nd Vehicular Technology Conference Fall 2010 (VTC 2010-Fall), Ottawa, Canada, Sep. 2010, pp. $1-5$.

[13] H. Urkowitz, "Energy detection of unknown deterministic signals," Proc. IEEE, vol. 55, no. 4, pp. 523-531, Apr. 1967. 